\title{
ORTHOCOMPACTNESS AND PERFECT MAPPINGS
}

\author{
DENNIS K. BURKE
}

\begin{abstract}
An example is given which shows that orthocompactness is not preserved by perfect maps. Subparacompact pointwise star-orthocompact spaces are orthocompact; this shows that orthocompactness is preserved by closed maps in the presence of subparacompactness.
\end{abstract}

A space $X$ is said to be orthocompact if every open cover $\mathcal{U}$ of $X$ has an open refinement $\mathcal{V}$ such that if $\mathcal{V}^{\prime} \subset \mathcal{V}$, then $\cap \mathcal{V}^{\prime}$ is open in $X$. Such a refinement $\mathfrak{V}$ of $\mathcal{U}$ is called a $Q$-refinement, and any open collecton $\mathscr{W}$ such that $\cap W^{\prime}$ is open whenever $W^{\prime} \subset W$ is called a $Q$-collection. The main purpose of this note is to provide an example showing the nonpreservation of orthocompactness under a perfect mapping, thus answering a question asked by $B$. Scott in $\left[\mathbf{S}_{1}\right]$ and $\left[\mathbf{S}_{2}\right]$. The reader is referred to these papers for an in-depth discussion of orthocompactness, especially the product theory.

The description of the example follows below. We use the convention that an ordinal number is the set of smaller ordinals, and $I$ denotes the "closed unit interval" from $R$. A mapping is a continuous onto function.

EXAMPLE 1. There exists an orthocompact space $X$ and a perfect mapping $f$ : $X \rightarrow Y$ onto a nonorthocompact space $Y$.

Proof. Let $X_{0}=\omega_{1} \times I \times\{0\}, X_{1}=\omega_{1} \times I \times\{1\}$, and $X=X_{0} \cup X_{1}$. For $\alpha, \beta \in \omega_{1}, \alpha$ a nonlimit ordinal with $\alpha \leqslant \beta, x \in I$, and $\varepsilon>0$ define

$$
\begin{aligned}
B(\alpha, \beta, x, \varepsilon)= & \left\{(\gamma, z, 0) \in X_{0}: \alpha<\gamma<\beta, 0<|x-z|<\varepsilon\right\} \\
& \cup\left\{(\gamma, z, 1) \in X_{1}: \alpha<\gamma<\beta,|x-z|<\varepsilon\right\} .
\end{aligned}
$$

Topologize $X$ by describing local bases as follows: Points $(\beta, x, 0) \in X_{0}$ are isolated in $X$. Points $(\beta, x, 1) \in X_{1}$ have the set of all $B(\alpha, \beta, x, \varepsilon)$, for nonlimit $\alpha<\beta$ and $\varepsilon>0$, for a local base. It may be revealing to the reader to provide a simple sketch here, and realize that $X$ is similar to, but not quite the same as, the "Alexandroff double" of $\omega_{1} \times I$.

To show $X$ is orthocompact, let $थ$ be an open cover of $X$. For each $x \in I$ consider $\mathcal{Q}$ as an open cover of $H_{x}=\omega_{1} \times\{x\} \times\{1\}$. There exists a nonlimit ordinal $\alpha_{x}<\omega_{1}$, an uncountable subset $A_{x} \subset\left[\alpha_{x}, \omega_{1}\right.$ ), and $\varepsilon_{x}>0$ (use $\varepsilon_{x}=1 / n$ for some appropriate positive integer $n$ ) such that for each $\beta \in A_{x}$ we have $B\left(\alpha_{x}, \beta, x, \varepsilon_{x}\right) \subset U$ for some $U \in \mathcal{Q}$. Note that the collection $\mathscr{W}_{x}=$ $\left\{B\left(\alpha_{x}, \beta, x, \varepsilon_{x}\right): \beta \in A_{x}\right\}$ is a $Q$-collection. For $x \in I$, let $J_{x}=\{z \in I:|x-z|<$ $\left.\varepsilon_{x}\right\}$; then $\mathcal{G}=\left\{J_{x}: x \in I\right\}$ is an open cover of $I$ so there is a finite set $F \subset I$ such

Received by the editors July 2, 1979 and, in revised form, August 29, 1979.

AMS (MOS) subject classifications (1970). Primary 54D20; Secondary 54C10, 54G20. 
that $I=\cup\left\{J_{x}: x \in F\right\}$. If $\beta_{0}=\max \left\{\alpha_{x}: x \in F\right\}$, the subspace

$$
Z=\left\{(\alpha, x, i): 0<\alpha<\beta_{0}, x \in I, i \in\{0,1\}\right\}
$$

is an open Lindelöf subspace of $X$, so there is an open cover $\mathcal{V}$ of $Z$ such that $\mathscr{V}$ is a $Q$-collection and each $V \in \mathcal{V}$ is contained in some $U \in \mathcal{Q}$. It follows that

$$
\mathscr{W}=\mathscr{V} \cup\left(\cup\left\{W_{x}: x \in F\right\}\right) \cup\left\{\{p\}: p \in X_{0}\right\}
$$

is a $Q$-cover of $X$ that refines $Q$.

Now let $Y=X_{0} \cup \omega_{1}$ and define a map $f: X \rightarrow Y: f(p)=p$ for $p \in X_{0}$ and $f(\alpha, x, 1)=\alpha$ for $(\alpha, x, 1) \in X_{1}$. Let $Y$ have the quotient topology induced by $f$. Clearly $f^{-1}(y)$ is compact in $X$ for each $y \in Y$ so to show $f$ is perfect it suffices to show $f$ is a closed mapping. Let $E \subset X$ be closed; to show $f(E)$ is closed in $Y$ we need only show that for any $\beta \in \omega_{1}-f(E)$ there is an open neighborhood $V$ of $\beta$ in $Y$ such that $V \cap f(E)=\varnothing$. Now $f^{-1}(\beta) \cap E=(\{\beta\} \times I \times\{1\}) \cap E=\varnothing$ so for each $x \in I$ there is $\delta_{x}>0$ and nonlimit $\alpha_{x} \leqslant \beta$ such that $B\left(\alpha_{x}, \beta, x, \delta_{x}\right) \cap E$ $=\varnothing$. Using the compactness of $I$, we see that there is some $r_{0}>0$, nonlimit $\gamma_{0}<\beta$, and a finite set $F \subset I$ such that

$$
\left(\cup\left\{B\left(\gamma_{0}, \beta, x, r_{0}\right): x \in F\right\}\right) \cap E=\varnothing
$$

and $\{\beta\} \times I \times\{1\} \subset \cup\left\{B\left(\gamma_{0}, \beta, x, r_{0}\right): x \in F\right\}$. Since $\cup\left\{B\left(\gamma_{0}, \beta, x, r_{0}\right): x \in\right.$ $F\}$ is saturated with respect to $f$, we have $V=f\left(\cup\left\{B\left(\gamma_{0}, \beta, x, r_{0}\right): x \in F\right\}\right)$ as the desired neighborhood of $\beta$ in $Y$ where $V \cap f(E)=\varnothing$.

To see that $Y$ is not orthocompact, we note that if $\beta \in \omega_{1}$ and $U \subset Y$ is open, with $\beta \in U$, there must be some nonlimit $\alpha<\beta$ such that $[\alpha, \beta] \subset U$ and $[\alpha, \beta] \times\{x\} \times\{0\} \subset U$ for all but finitely many $x \in I$. Let $B=\left\{z_{\alpha}: \alpha<\omega_{1}\right\}$ be a subset of $I$, indexed by $\omega_{1}$, where $z_{\alpha} \neq z_{\beta}$ if $\alpha \neq \beta$. For each $\beta<\omega_{1}$ let $G_{\beta}=f\left(B\left(0, \beta, z_{\beta}, 1\right)\right)$ and $\mathcal{G}=\left\{G_{\beta}: \beta<\omega_{1}\right\}$; then $\mathcal{G}$ is an open cover of $Y$ and if $\mathcal{H}$ is any open refinement of $\mathcal{G}$ there is some $\gamma \in \omega_{1}$ such that $\left[\gamma, \omega_{1}\right) \subset \operatorname{St}(\gamma, \mathcal{H})$. This can happen only if there is an uncountable set $A \subset\left[\gamma, \omega_{1}\right)$ where for each $\beta \in A$ there is $H_{\beta} \in \mathcal{H}$ such that $\gamma \in H_{\beta} \subset G_{\beta}$. It follows that

$$
\left(\bigcap_{\beta \in A} H_{\beta}\right) \cap\left(\omega_{1} \times\left\{z_{\alpha}\right\} \times\{0\}\right)=\varnothing
$$

for every $\alpha \in A$, hence $\gamma \notin \operatorname{int}\left(\cap_{\beta \in A} H_{\beta}\right)$ and $\mathcal{H}$ cannot be a $Q$-refinement of $\mathcal{G}$. That concludes the verification of the stated properties of Example 1.

The proof, in the above example, that $Y$ is not orthocompact, was given for completeness. Other authors have considered similar examples and results which essentially show the nonorthocompactness of $Y$. G. Gruenhage [G] gave an example of a nonorthocompact space which is the closed image of an orthocompact space. Gruenhage's range space is homeomorphic to a closed subspace of $Y$ (and, under $\mathrm{CH}$, is homeomorphic to $Y$ ) and certainly Gruenhage's result implies the nonorthocompactness of $Y$. The essential reason for the nonorthocompactness of $Y$ can also be culled from results in $\left[\mathbf{S}_{1}\right]$ or $\left[\mathbf{S}_{2}\right]$, which show that $\omega_{1} \times\left(\omega_{1}+1\right)$ is not orthocompact. For other related results on the construction of nonorthocompact spaces the reader is referred to [HL]. 
The existence of Example 1 increases the importance of several generalizations of orthocompactness, considered by other authors, that are preserved under closed or perfect mappings. Weakly orthocompact spaces $\left[\mathbf{S}_{1}\right]$ are preserved under perfect maps, discretely orthocompact spaces $[\mathbf{J}]$ are preserved under closed mappings, and pointwise star-orthocompact spaces [G] are preserved under closed mappings. These concepts are useful in helping to preserve orthocompactness, under closed maps, when in the presence of other covering properties. Junnila [J] has shown that a $\theta$-refinable space $X$ is orthocompact if it is discretely orthocompact (see [J] for definition) and as a corollary he obtains:

THEOREM 2 [J]. If $f: X \rightarrow Y$ is a closed continuous onto map, and $X$ is a $\theta$-refinable orthocompact space, then so is $Y$.

A somewhat weaker result can be obtained via the pointwise star-orthocompactness defined by Gruenhage [G]. A space $X$ is pointwise star-orthocompact if for any open cover $\mathcal{Q}$ of $X$ there is a $Q$-collection $\left\{V_{x}: x \in X\right\}$ such that $x \in V_{x} \subset$ $\operatorname{St}(x, \mathcal{U})$ for each $x \in X$. Gruenhage shows that any pointwise star-orthocompact developable space is orthocompact; a modification of Gruenhage's proof actually yields the following stronger result.

THEOREM 3. If $X$ is a subparacompact pointwise star-orthocompact space then $X$ is orthocompact.

Proof. If $\mathscr{U}$ is an open cover of the subparacompact space $X$ there is a sequence $\left\{\mathcal{G}_{n}\right\}_{1}^{\infty}$ of open covers of $X$ such that if $x \in X$ there is some $n \in N$ (depending on $x$ ) such that $\operatorname{St}\left(x, \mathcal{G}_{n}\right) \subset U$ for some $U \in \mathcal{U}$ (see [B]). Apply pointwise star-orthocompactness to each $\mathcal{G}_{n}$ and it follows that $\mathcal{U}$ has an open refinement which is the union of a countable number of $Q$-collections. Since a subparacompact space is countably metacompact, we see that $X$ is orthocompact $\left[\mathbf{S}_{\mathbf{1}}\right]$.

Since subparacompactness is preserved under closed maps [B], we have the weaker version of Theorem 2 , using "subparacompact" in place of " $\theta$-refinable".

\section{REFERENCES}

[B] D. Burke, On subparacompact spaces, Proc. Amer. Math. Soc. 23 (1969), 655-663.

[G] G. Gruenhage, On closed images of orthocompact spaces, Proc. Amer. Math. Soc. 77 (1979), 389-394.

[HI] R. W. Heath and W. F. Lindgren, On generating nonorthocompact spaces, Set-Theoretic Topology, Academic Press, New York, 1977, pp. 225-237.

[J] H. Junnila, Covering properties and quasi-uniformities of topological spaces, Doctoral dissertation, Virginia Polytechnic Inst. and State Univ., 1978.

[S $\mathbf{S}_{1}$ B. M. Scott, Toward a product theory for orthocompactness, Studies in Topology, Academic Press, New York, 1975, pp. 517-537.

[S $\left.\mathrm{S}_{2}\right]$ More about orthocompactness (to appear).

Department of Mathematics, MuMi University, OXPORD, OHo 45056 